\title{
Twelve-Weeks of Bench-Step Exercise Training Ameliorates Cardiopulmonary Fitness and Mood State in Patients with Schizophrenia: A Pilot Study
}

\author{
Yu-Chi Kuo, Dan-Yan Chang and Yi-Hung Liao * \\ Department of Exercise and Health Science, National Taipei University of Nursing and Health Sciences, \\ Taipei 11219, Taiwan; yuchi.kuo@gmail.com (Y.-C.K.); helloimden@gmail.com (D.-Y.C.) \\ * Correspondence: yihungliao.henry@gmail.com; Tel.: +86-2-2822-7101 (ext. 7707)
}

Citation: Kuo, Y.-C.; Chang, D.-Y.;

Liao, Y.-H. Twelve-Weeks of

Bench-Step Exercise Training Ameliorates Cardiopulmonary Fitness and Mood State in Patients with Schizophrenia: A Pilot Study. Medicina 2021, 57, 149. https:// doi.org/10.3390/medicina57020149

Academic Editor: Simon M. Fryer

Received: 7 January 2021

Accepted: 4 February 2021

Published: 7 February 2021

Publisher's Note: MDPI stays neutral with regard to jurisdictional claims in published maps and institutional affiliations.

Copyright: (c) 2021 by the authors. Licensee MDPI, Basel, Switzerland. This article is an open access article distributed under the terms and conditions of the Creative Commons Attribution (CC BY) license (https:// creativecommons.org/licenses/by/ $4.0 /)$.

\begin{abstract}
Background and objectives: Unhealthy, physically inactive lifestyles increase the risk of future cardiovascular events and impaired physical fitness in individuals with schizophrenia. Insufficient literature exists to provide fundamental information about appropriate exercise training modality for this population. This pilot study preliminarily investigated the effects of a 12-week moderate-intensity bench-step exercise training (BSET) program on cardiopulmonary fitness, mood state, and cognition in patients with schizophrenia. Methods: Twenty-eight patients with schizophrenia completed this study. The participants were allocated into either bench-step exercise-training (BSET; $N=14)$ or control (CTRL; $N=14$ ) groups according to their preferences. The BSET group received a 12-week bench-step intervention, whereas the CTRL group did not participate in any training. The Beck Depression Inventory-II (BDI-II), 6-min walk test (6MWD), and Symbol Digit Modalities Test (SDMT) were assessed at baseline (PRE) and at the end of the intervention (POST) to determine mood state, endurance fitness, and attention, respectively. Results: After a 12-week BSET intervention, the 6MWD was significantly increased in the BSET $(p=0.007)$ but not in the CTRL $(p>0.05)$. The participants with BSET intervention showed a significant decrease in BDI-II at the end of the intervention $(p=0.03)$. However, SDMT scores were not different in both BSET and CTRL $(p>0.05)$. Conclusions: This study demonstrated that the 12-week intervention of moderate-intensity bench-step exercise training (frequency: 1 session/week; each session of $30 \mathrm{~min}$; step cadence: 96 beats/min) might effectively enhance cardiopulmonary fitness and mood state in patients with schizophrenia. However, attention did not change after the bench-step exercise intervention.
\end{abstract}

Keywords: 6-min walk distance (6MWD); beck depression inventory-II (BDI-II); symbol digit modalities test (SDMT); mood states; fitness; attention

\section{Introduction}

Evidence indicates that 1 in 100 individuals may develop a mental disorder at a certain point in their life [1]. Currently, the global prevalence of mental disorders is approximately $1 \%$, with an incidence rate of $0.02-2.16$ per 1000 individuals [2]. Of these, approximately 250 million individuals have schizophrenia [3]. Schizophrenia is characterized by thinking and cognitive impairments and behavioral and psychiatric symptoms such as hallucinations (auditory or visual) or delusions (persecutory or reference). As the disease progresses, patients with schizophrenia develop chronic symptoms, including persistent negative symptoms and cognitive impairments, often resulting in a permanent disability that burdens the patients and their family members as well as the healthcare system [3].

Several unhealthy lifestyles, including sedentary behavior or physical inactivity, have commonly been observed in individuals with schizophrenia. Such behavioral features could lead to the rapid development of future cardiovascular events and metabolic disorders [4-7]. Antipsychotics are commonly used for controlling the related symptoms in 
individuals with schizophrenia [8]. It has been reported that the use of antipsychotics is associated with the prevalence of metabolic disorders $[9,10]$. More importantly, there is a higher prevalence of diabetes mellitus [11,12], overweight and metabolic diseases [13], and cardiovascular respiratory diseases $[14,15]$ in the population with schizophrenia.

Cognitive impairments perturb patients with schizophrenia from living independently. Studies have employed neuropsychological testing to verify that these patients have general cognitive impairments [16]. Moreover, impairment in attention/concentration in patients with schizophrenia also results in impeding them from learning complex work skills $[17,18]$. Patients with schizophrenia may develop obesity-related degenerative disorders (e.g., diabetes, cardiovascular diseases, hypertension, and other chronic diseases) due to unhealthy living behaviors (e.g., smoking, alcohol consumption, and sedentary lifestyle) as well as side effects from antipsychotic agents. These factors all potentially reduce their cardiopulmonary fitness and increase their mortality by two to three times compared with healthy general individuals [19-22]. In this regard, it would be important for clinical professionals to develop effective non-pharmaceutical practices to better attenuate these negative impacts from schizophrenia.

Exercise training can enhance the physical fitness of patients with schizophrenia. For instance, Scheewe et al. (2013) reported that participants with schizophrenia enrolled in muscle endurance exercise training enhanced their cardiopulmonary endurance and quality of life (QoL). Additionally, the training alleviated their negative symptoms, depression, and anxiety [23]. Acute high-intensity exercise has been reported to show worse cognitive performance compared with moderate-intensity exercise and sedentary controls [24,25]. For relative lower intensity exercise, exercise duration may be extended after patient safety and experience are considered [26]. For example, McDevitt et al. administered 12-week walking exercise training (three sessions weekly, each session of $60 \mathrm{~min}$ ) to patients with severe mental disorders and found that this intervention enhanced patients' emotional and social function and that adequate exercise could ameliorate negative symptoms in patients with schizophrenia [27]. The relationship between cognitive function and exercise intensity can be represented as an inverted U-shaped curve [25], suggesting that the use of moderately intense exercise could better enhance cognitive function in patients with schizophrenia.

Taken together, the benefits of exercise on physiological and psychological health have been well recognized. Although exercise in clinical practice is currently available for patients with schizophrenia, studies on relevant routine exercise interventions with fixed intensity and desirable accessibility have been scant. Most of the aforementioned relevant studies were based on relatively low-intensity exercises (e.g., walking exercise training) $[23,27]$. Consequently, these exercises may be insufficient for fulfilling the treatment need for the moderate-intensity exercise of patients with schizophrenia [26]. More importantly, however, high-intensity aerobic training (85-95\% heart rate reserve [HRR]) leads to reduced patient participation rates with schizophrenia ( $24 \%$ attrition rate) [28]. Therefore, we proposed this pilot study to preliminarily investigate the effects of a 12-week moderate-intensity bench-step exercise training (BSET; frequency: 1 session/week; each session of $30 \mathrm{~min}$; step cadence: 96 beats/min) on cardiopulmonary fitness, mood state, and cognition in patients with schizophrenia. We hypothesized that a 12-week intervention of bench-step exercise can effectively alleviate psychiatric syndromes and enhance aerobic capacity in patients with schizophrenia.

\section{Materials and Methods}

\subsection{Participants and Ethical Statement}

This study recruited thirty patients diagnosed as having chronic schizophrenia from a community halfway house. Subsequently, we grouped the participants into bench-step exercise-training (BSET) and control (CTRL) groups according to their preference. The reason to allocate the participants was based on their preference due to the clinical condition with chronic schizophrenia, thus we were not to be able to perform the randomized 
allocation in this study. After the recruitment, there were 30 eligible participants enrolled in this study, and each group was comprised of 15 participants. During the intervention, there was one participant from each group that dropped out of the study due to personal reasons. All exercise interventions were supervised by a registered occupational therapist, and only participants who attended all twelve times training (frequency: once a week) were included in the final analysis, thus the exercise training compliance ratio was $100 \%$. For the drop-out ratio, one from each group dropped out due to their personal reason during the intervention, thus the drop-out ratio was $6.67 \%$ ( 2 of 30 participants). In total, 28 participants completed the study. The participants were included only if they: (1) were aged 20-65 years; (2) diagnosed as having schizophrenia according to the Diagnostic and Statistical Manual of Mental Disorder, Fifth Edition, Text Revision (DSM-V-TR; APA, 2013); and (3) had diagnosed schizophrenia for 2 years or more. In brief, the DSM-V-TR diagnosed criteria for schizophrenia include: (i) delusions; (ii) hallucinations; (iii) disorganized speech; (iv) grossly disorganized or catatonic behavior; and (v) negative symptoms, and two or more of the above symptoms ( $>1$ month) and at least one of them must be (i), (ii), or (iii). Moreover, some signs of the above disorder must last for a continuous period of at least 6 months for the DSM-V-TR standards. The participants were excluded if they: (1) were unable to follow verbal commands or perform exercise training (both indicating considerable interference in language, cognitive, behavioral, and mental conditions); and (2) had a history of epilepsy, heart disease, and pulmonary function impairment and untreated hypertension. All participants completed a written informed consent form (ICF) and a self-screening health questionnaire (Physical Activity Readiness Questionnaire, PARQ) before enrolling in the study. For the procedure of signing written informed consent, in addition to the researcher explaining the entire study process, the research crew members also asked the potential participants to repeat explaining the process to ensure that the potential participants understood all of the experimental procedures and what they need to cooperate during the intervention. This study was conducted with the approval of the National Taiwan University Research Ethics Committee (NTU-REC No: 201703EM012; date: 9 May 2017).

\subsection{Experiment Design and Procedure}

The BSET group received a 12-week intervention of bench-step exercise training, whereas the CTRL group did not receive any type of exercise-training. One day before the intervention, the participant's baseline characteristics, including height, weight, blood pressure, and heart rate, and Beck Depression Inventory-II (BDI-II) score and Symbol Digit Modalities Test (SDMT) were acquired in the pretest (PRE). Moreover, for the cardiopulmonary/endurance fitness assessment, we instructed the participants to perform a 6-min walking test (6MWD). At the end of the intervention, the aforementioned values were measured again in a posttest (POST). The POST was performed one day after the last intervention to avoid possible influence from the previous exercise session. All test procedures were identical between pretest (PRE) and posttest (POST).

\subsection{Bench-Step Exercise Training}

Our 12-week bench-step exercise training was adapted from a previous study [29] with minor modifications with adjusting the time of warm-up and cool-down exercise. An entire training session lasted $30 \mathrm{~min}$. The sessions were conducted weekly for 12 weeks. In brief, before each training session started and ended, the participants were asked to perform 5-min warm-up and 5-min cool-down exercises. These exercises included 5 min of stretching exercises, static stretching exercises (each movement lasted for $10 \mathrm{~s}$; stretching parts: head, neck, spine, and upper and lower limb) were used, and the stretching movements included flexion, extension, adduction, abduction, rotation, and circumduction. After completing the warm-up exercises, the participants performed 20-min bench-step exercise training beginning at 96 beats / $\mathrm{min}$ on a 10-cm-high step (24 sets of stepping up and down the step), which had been shown to elicit an intensity of 40-60\% HRR [29]. 
For every participant, the exercise intensity was increased when their exercise capacity improved. During each training session, the trainer consistently asked the participants' subjective rate of perceived exertion (RPE; 0-10 scale), and their perceived effort should be within the RPE range of 3-4 to ensure the target intensity (equivalent to $40-60 \%$ HRR) were reached. According to the participants' real-time perceived effort responses, the exercise intensity was personalized by the trainer's vocal guidance to change their arm-swinging frequencies to reach the appropriate training pace.

\subsection{Six-Minute Walk Distance Test (6MWD)}

The 6-min walking distance test (6MWD) is commonly used to determine whether a participant can complete daily tasks in their living environments. Previous studies have indicated that the $6 \mathrm{MWD}$ provides reliable data on physical performance and the clinical functionality of patients with schizophrenia. The 6MWD is capable of rapidly evaluating the effects of moderate- and high-intensity exercises on these patients $[6,7,30,31]$. Moreover, the $6 \mathrm{MWD}$ is a cost-effective, simple, convenient, and rapid test for evaluating the functional performance of patients with schizophrenia [30]. In clinical practice, the $6 \mathrm{MWD}$ is used to evaluate aerobic endurance according to the standard procedures detailed by Stookey et al. [32]. Here, our participants were asked to walk at their highest speed possible on a 100-foot-long flat ground surface for continuous $6 \mathrm{~min}$. During the 6MWD test, a research crew guided the participant in completing the test by providing accurate instructions and positive feedback.

\subsection{Symbol Digit Modalities Test (SDMT)}

Symbol Digit Modalities Test (SDMT) is a written test to assess cognitive functioning [33]. The participants were instructed to pair a series of eight symbols with their corresponding numerical digits using a key at the top of the test page. In brief, the participants were required to fill in the boxes under each symbol with their corresponding numerical digit. The total number of these boxes was 110 . The test duration was $90 \mathrm{~s}$. The number of items answered correctly within these $90 \mathrm{~s}$ indicated the participant's processing speed. Because SDMT test provided hints to reduce the amount of participant memory required for answer memorization. Therefore, SDMT mainly assessed the selective attention of the participants in this present investigation.

\subsection{Beck Depression Inventory-II (BDI-II)}

BDI-II is a self-reported depression inventory [34]. The participants were asked to score 21 depression-related items on a 4-point scale (0-3). Those who scored 0-13, 14-19, 20-28, and 29-63 points were determined to have minimal, mild, moderate, and severe depression, respectively. In clinical practice, BDI-II is considered to reflect only the degree of depression and thus is not used as a depression diagnosis instrument. However, the BDI-II manual suggests that this inventory reflects the cognitive and emotional status of patients with depression, as well as their somatic and vegetative status.

\subsection{Statistical Analysis}

The commercially available statistical software SPSS 20.0 (version 20.0; IBM Corp., Armonk, NY, USA) was used for statistical analysis. The normality of all measured variables was analyzed using the Shapiro-Wilk normality test provided by SPSS 20.0 software. The independent sample $t$-test and the chi-square test were used to compare the baseline characteristics between both groups. A two-way analysis of variation (2-way ANOVA) was subsequently employed to compare the SDMT, 6MWD, and BDI-II results between both groups. Post hoc analysis using the Bonferroni correction was adopted to detect interactions if the interaction was significant or the parameters under the same categories were compared. The statistical significance level was set at $\alpha=0.05$, and all statistical data are presented as means \pm standard deviations. The effect size $\left(\eta^{2}\right)$ are categorized as small: 
$<0.01$; medium: 0.06; or large: $>0.14$. The effect size (Cohen's d) are categorized as small: $0.2-0.3$; medium: $\approx 0.5$; or large: $\geq 0.8$.

\section{Results}

\subsection{Basic Characteristics of Participants}

All of the population characteristics and distribution are shown in Table 1. The gender distribution was the same for both males and females (50\% each). The SDMT scores were mostly distributed at the subgroup range with $21-30$ and $31-41$ scores. The BDI-II score was distributed within 0-13, and the 6MWD distance ranged within 375-449.5 $\mathrm{m}(11,39.3 \%)$. The basic characteristics of the participants are displayed in Table 1 . The average participant age was $52.1 \pm 11.0$ years old (BSET) and $50.1 \pm 8.3$ years old (CTRL). The average participant BMI was $24.7 \pm 4.6 \mathrm{~kg} / \mathrm{m}^{2}$ (BSET) and $24.7 \pm 3.8 \mathrm{~kg} / \mathrm{m}^{2}$ (CTRL). The BDI-II assessment score at participant baseline was $11.3 \pm 11.6$ (BSET) and $10.9 \pm 9.5$ (CTRL); moreover, the SDMT attention assessments did not show a difference between both groups (BSET: $30.7 \pm 9.8$ tasks; CTRL: $33.2 \pm 10.7$ tasks). The distance of $6 \mathrm{MWD}$ was $402.4 \pm 78.9 \mathrm{~m}$ (BSET) and $418.1 \pm 55.3 \mathrm{~m}$ (CTRL). There were no significant differences between the BSET group and CTRL group in all baseline characteristics.

Table 1. Basic population characteristic distribution of all participants.

\begin{tabular}{|c|c|c|c|c|}
\hline Variable & $\begin{array}{c}\text { Total Number } N \\
(\%)\end{array}$ & $\underset{(\%)}{\text { BSET Group } N}$ & $\underset{(\%)}{\text { CTRL Group } N}$ & $p$-Value \\
\hline Number & $28(100 \%)$ & $14(50 \%)$ & $14(50 \%)$ & \\
\hline Height (cm) & - & $168.1 \pm 7.7$ & $164.4 \pm 7.9$ & 0.111 \\
\hline Body weight (kg) & - & $69.4 \pm 10.9$ & $67.1 \pm 14.6$ & 0.324 \\
\hline Gender & & & & 0.1 \\
\hline Male & $14(50 \%)$ & $9(64.3 \%)$ & $5(35.7 \%)$ & \\
\hline Female & $14(50 \%)$ & $5(35.7 \%)$ & $9(64.3 \%)$ & \\
\hline Age (yrs) & - & $52.1 \pm 11.0$ & $50.1 \pm 8.3$ & 0.296 \\
\hline $21-30$ yrs & $1(3.6 \%)$ & $1(7.1 \%)$ & $0(0 \%)$ & \\
\hline $31-40$ yrs & $2(7.1 \%)$ & $1(7.1 \%)$ & $1(7.1 \%)$ & \\
\hline $41-50$ yrs & $9(32.1 \%)$ & $3(21.4 \%)$ & $6(42.9 \%)$ & \\
\hline $51-60$ yrs & $10(35.7 \%)$ & $5(35.7 \%)$ & $5(35.7 \%)$ & \\
\hline $61-65$ yrs & $6(21.4 \%)$ & $4(28.6 \%)$ & $2(14.3 \%)$ & \\
\hline BMI $\left(\mathrm{kg} / \mathrm{m}^{2}\right)$ & - & $24.7 \pm 4.6$ & $24.7 \pm 3.8$ & 0.489 \\
\hline $18 \leq \mathrm{BMI}<24$ & $12(42.9 \%)$ & $6(42.9 \%)$ & $6(42.9 \%)$ & \\
\hline $24 \leq \mathrm{BMI}<31$ & $16(57.1 \%)$ & $8(57.1 \%)$ & $8(57.1 \%)$ & \\
\hline SDMT̄̄ (task scores) & - & $30.7 \pm 9.8$ & $33.2 \pm 10.7$ & 0.252 \\
\hline $11-20$ scores & $3(10.7 \%)$ & $1(7.1 \%)$ & $2(14.3 \%)$ & \\
\hline $21-30$ scores & $10(35.7 \%)$ & $6(42.9 \%)$ & $4(28.6 \%)$ & \\
\hline $31-40$ scores & $10(35.7 \%)$ & $5(35.7 \%)$ & $5(35.7 \%)$ & \\
\hline $41-50$ scores & $3(10.7 \%)$ & $1(7.1 \%)$ & $2(14.3 \%)$ & \\
\hline $51-60$ scores & $2(7.1 \%)$ & $1(7.1 \%)$ & $1(7.1 \%)$ & \\
\hline BDI-II (score) & - & $11.3 \pm 11.6$ & $10.9 \pm 9.5$ & 0.459 \\
\hline 0-13 Minimal & $17(60.7 \%)$ & $9(64.3 \%)$ & $8(57.1 \%)$ & \\
\hline 14-19 Mild & $3(10.7 \%)$ & $0(0 \%)$ & $3(21.4 \%)$ & \\
\hline 20-28 Moderate & $7(25 \%)$ & $4(28.6 \%)$ & $3(21.4 \%)$ & \\
\hline 29-63 Severe & $1(3.6 \%)$ & $1(7.1 \%)$ & $0(0 \%)$ & \\
\hline 6MWD (meters) & - & $402.4 \pm 78.9$ & $418.1 \pm 55.3$ & 0.266 \\
\hline$<300 \mathrm{~m}$ & $1(3.6 \%)$ & $1(7.1 \%)$ & $0(0 \%)$ & \\
\hline $301-374.9 \mathrm{~m}$ & $7(25 \%)$ & $3(21.4 \%)$ & $4(28.6 \%)$ & \\
\hline $375-449.5 \mathrm{~m}$ & $11(39.3 \%)$ & $5(35.7 \%)$ & $6(42.9 \%)$ & \\
\hline$>450 \mathrm{~m}$ & $9(32.1 \%)$ & $5(35.7 \%)$ & $4(28.6 \%)$ & \\
\hline
\end{tabular}

BMI, body mass index; SDMT, Symbol Digit Modalities Test; BDI-II, Beck Depression Inventory-II; 6MWD, Six-Minute Walk Distance Test.

\subsection{MWD Endurance Performance after BSET Training}

The walking distance $(\mathrm{m})$ for the 6-min walking distance (6MWD) has been widely used as the cardiorespiratory fitness/endurance index. This also reflects the capacity for 
conducting daily life activities. The 6MWD results are displayed in Table 2 . The walking distance for the 6MWD was measured at baseline (PRE) and after the intervention (POST). There was no interaction between the two groups, but there was a time effect (power $=0.156$; $F(1,28)=0.951 ; \eta^{2}=0.033$, small-to-medium $\left.E S ; p<0.05\right)$. We further compared the differences between PRE and POST within each group and found that, after 12-week bench-step exercise training intervention, the 6MWD distance performance was significantly increased in the BSET group ( $p=0.007$; Cohen's d effect size $=0.424$, medium $E S$ ) but not in the CTRL group $(p>0.05)$.

Table 2. Cardiopulmonary fitness, mood state, and attention.

\begin{tabular}{|c|c|c|c|c|c|c|c|c|}
\hline Item & Group & $\begin{array}{c}\text { PRE } \\
\text { (Baseline) }\end{array}$ & $\begin{array}{l}\text { POST } \\
\text { (End) }\end{array}$ & Time & Treatment & Interaction & $\begin{array}{c}\text { within } \\
p \text {-Value }\end{array}$ & $\begin{array}{c}\text { Cohen's } \\
d E S\end{array}$ \\
\hline \multirow[t]{2}{*}{ 6MWD (meters) } & BSET & $402.4 \pm 78.9$ & $436.4 \pm 81.5^{*}$ & $p<0.01$ & $p=0.88$ & $p=0.15$ & $p<0.01$ & 0.424 \\
\hline & CTRL & $418.1 \pm 55.3$ & $428.4 \pm 71.8$ & & (n.s.) & (n.s.) & $p=0.18$ & 0.160 \\
\hline \multirow{2}{*}{ BDI-II (scores) } & BSET & $11.3 \pm 11.6$ & $8.8 \pm 9.6^{*}$ & $p<0.05$ & $p=0.85$ & $p=0.14$ & $p=0.03$ & 0.235 \\
\hline & CTRL & $10.9 \pm 9.5$ & $10.6 \pm 8.7$ & & $($ n.s. $)$ & (n.s.) & $p=0.31$ & 0.032 \\
\hline \multirow[t]{2}{*}{ SDMT (tasks) } & BSET & $30.7 \pm 9.8$ & $33.6 \pm 11.7$ & $p=0.07$ & $p=0.72$ & $p=0.27$ & $N / A$ & $N / A$ \\
\hline & CTRL & $33.2 \pm 10.7$ & $33.9 \pm 12.1$ & (n.s.) & $(n . s)$. & (n.s.) & $N / A$ & $N / A$ \\
\hline
\end{tabular}

* denotes the difference between PRE and POST within the group $(p \leq 0.05)$. ES, effect size; SDMT, Symbol Digit Modalities Test; BDI-II, Beck Depression Inventory-II; 6MWD, Six-Minute Walk Distance Test; $n$.s., non-significance within group $(p>0.05)$.

\subsection{The BSET Training Effects on Mood State (BDI-II) and Attention (SDMT)}

The mood of patients with chronic schizophrenia was tested using the Beck Depression Inventory-II (BDI-II) (Table 2). There was a time effect (power $=0.487 ; F(1,28)=3.985$; $\eta^{2}=0.125$, medium-to-large $\left.E S ; p<0.05\right)$ but not a treatment effect $(p>0.05)$ in BDI-II. The participants with BSET training intervention showed a decrease in BDI-II from $11.4 \pm 12$ points at baseline (PRE) to $8.8 \pm 9.6$ points at the end of the intervention (POST) $\left(p=0.03 ; \mathrm{Co}^{-}\right.$ hen's d effect size $=0.235$, small ES). However, there were no differences in BDI-II after the intervention (PRE: $10.9 \pm 9.5$ points vs. POST: $10.6 \pm 8.7$ points) compared to the CTRL group $(p>0.05)$. On the other aspect, after 12 weeks of BSET intervention, the corrected task number of symbolic digital module test (SDMT) scores were not statistically different between the two different test stages in both the BSET group and CTRL group $(p>0.05)$ (Table 2).

\section{Discussion}

We administered a 12-week moderate-intensity bench-step exercise training intervention, including 30-min sessions performed once weekly. The primary findings of this study are that 12-weeks endurance exercise training effectively enhanced their mood state and cardiopulmonary fitness/endurance, measured using 6MWD, in patients with chronic schizophrenia. However, the attention level did not exhibit significant enhancements after the exercise intervention compared with the pretest baseline values.

The main objective of schizophrenia treatment is to improve the functional prognosis [35]. Recent clinical research on schizophrenia has focused on metabolic health enhancement and the alleviation of cardiovascular comorbidities [15,36-38]. Patients with schizophrenia have been reported to have an increased rate of premature mortality due to cardiovascular comorbidities [39]. Epidemiological evidence reveals that individuals with schizophrenia exhibited three times the risk of suffering sudden cardiac death than those from the general population [40,41]. Additionally, patients with schizophrenia receiving antipsychotics exhibited a very high incidence of metabolic disorders and increased cardiovascular risk [42]. The correlation between schizophrenia and metabolic and cardiovascular diseases mainly involves complex environmental and health factor interactions (i.e., physical activity, acquired nutrition, and drug use) [19-22]. Hence, it has been suggested that regularly re-evaluating the cardiovascular risk and initiation of behavioral changes are critical to maintaining the health status in the schizophrenia population [43]. 
Vancampfort et al. (2011) indicated that the amount of physical activity (e.g., walking distance) during leisure time is positively correlated to the health-related QoL of patients with schizophrenia [7]. Therefore, assessing exercise capability is crucial to understanding the effects of schizophrenia and developing healthcare management methods for patients with schizophrenia $[6,7,31,44]$. Studies indicated that the $6 \mathrm{MWD}$ provides data on physical performance and clinical functions of patients with schizophrenia and thereby serves as an effective, simple, convenient, and rapid test to evaluate the effects of moderate- and highintensity exercises on these patients $[6,7,30,31]$. In addition, patients with schizophrenia with regular exercising habits and without any metabolism disorder have long walking distances on the 6MWD [44]. Notably, in this study, we employed the 6MWD to evaluate the aerobic capacity of patients with schizophrenia and found that our 12-week intervention enhanced the patients' aerobic endurance and fitness effectively. Therefore, weekly 30-min sessions of moderate- to high-intensity exercises can enhance the aerobic endurance and exercise capabilities of patients with schizophrenia. Moreover, several lines of evidence reveal that people with schizophrenia are less active and exercise less than those from the general population $[4,6,7]$. In this regard, because physical exercise capabilities and health-related QoL have been confirmed to be correlated positively [7], we suggest that patients with schizophrenia should conduct at least $30 \mathrm{~min}$ of moderate- to high-intensity exercises every week to alleviate cardiovascular and metabolic disorders and maintain physical health.

Empirical intervention studies have proven and supported the benefits of exercise in patients with mental disorders $[45,46]$. A comprehensive analytical study revealed that weekly interventions of moderate- to high-intensity exercises for $90 \mathrm{~min}$ significantly alleviated psychiatric symptoms [46]. Donaghy reviewed 11 prospective longitudinal studies and revealed that exercise training is an effective intervention strategy to mitigate depression. In addition, exercise is a complementary treatment strategy for mild and moderate depression and can serve as a nonpharmacological treatment method to enhance cognitive functioning [45]. Most studies have supported that exercise training has positive effects in patients with schizophrenia: it enhances cognitive function and alleviates depressive psychiatric symptoms [45-48]. However, a study has revealed that high-intensity (85-95\% HRR) aerobic exercise may reduce the participation rate of patients with severe schizophrenia [28]. Heggelund et al. recruited 25 younger patients with schizophrenia (mean age $=27$ years) to participate in an eight-week intervention of high-intensity interval aerobic training (three sessions weekly, each session of $25 \mathrm{~min}$ ), but six of their participants dropped out (attrition rate $=24 \%$ ) [28]. Of note, we observed that none of the participants in the bench-step exercise group dropped out during the 12-week intervention, suggesting a relatively low-frequency training program could be effective to simultaneously alleviate cardiopulmonary fitness, attenuate depression levels, and improve the adhesion for training in patients with schizophrenia.

Our results indicate that our 12-week intervention of moderate-intensity (once per week) bench-step exercise training effectively improved overall depression level (reduced BDI-II score; Table 2). Our result is in line with previous studies that exercise training is capable of improving psychiatric symptoms of patients with schizophrenia $[28,30,45,46,48]$. For example, Vancampfort et al. (2012) discovered that lower 6MWD scores are correlated with negative and depressive psychiatric symptoms [30]. Moreover, another study employed an eight-week intervention of high-intensity interval aerobic training (three sessions weekly, each session of $25 \mathrm{~min}$; the total number of sessions $=24$, average weekly training duration $=72 \mathrm{~min}$ ) alleviated the depressive symptoms of patients with schizophrenia [28].

However, in this study, the attention, measured by SMDT, did not exhibit overall improvements after bench-step exercise intervention. Possible reasons for this discrepancy include our relatively low sample size and relatively short intervention duration. Previous studies in which patients with schizophrenia achieved benefits on cognition employed higher training intensities and longer training durations (72-180 min weekly). For instance, Kimhy et al. adopted a 12-week intervention of moderate-intensity training (three sessions 
weekly, each session of $60 \mathrm{~min}$; the total number of sessions $=36$, average weekly training duration $=180 \mathrm{~min}$ ) and reported significant improvements in aerobic fitness and MATRICS Consensus Cognitive Battery scores of patients with schizophrenia [48]. Therefore, future studies may include low-intensity exercise over longer intervention periods to verify whether this type of intervention can alleviate cognitive functions further.

SDMT total scores represent the participants' selective attention capacity. However, in our exercise-training group, these scores did not exhibit significant differences compared with that of the control group. Stroth et al. (2009) reported that continuous aerobic exercise for 6 weeks (three sessions weekly, each session of $30 \mathrm{~min}$ ) significantly enhanced the memory-related cognitive function performance in generally healthy adults [49]. Thus far, few intervention studies have evaluated the influence of endurance training on attention in patients with schizophrenia [50,51]. Malchow et al. (2015) employed three months of bundle intervention that included low-intensity cycling endurance training (lactic acid concentration of 1.5-2 mmol/L; three sessions weekly, each session of $30 \mathrm{~min}$ ) and computer-assisted cognitive remediation (CACR) (two sessions weekly, each session of $30 \mathrm{~min}$; initiated at the sixth week of training) [50]. Their results revealed that, between the sixth week and the final day of the three-month intervention, the cognitive function performance of patients with schizophrenia who received bundle intervention (i.e., endurance training and CACR) was enhanced significantly [50]. Despite the training intensity employed in this study is higher than that used by Malchow et al. [50], we did not observe a significant enhancement in cognitive function. This is possibly because Malchow et al. (2015) included additional CACR intervention, which might further enhance training diversity and complexity. Moreover, the training frequencies and total weekly training durations differed between our present study and that of Malchow et al. (2015). This indicates that the employed exercise volume to a total of $90 \mathrm{~min} /$ week could significantly enhance the neurocognitive function of patients with schizophrenia [46]. Therefore, a more diverse and greater training volume, as used by Malchow et al. (2015), can enhance the cognitive function of patients with schizophrenia further.

There are still some limitations to this pilot study. First, we allocated the participants into their treatment group based on their preference but not randomization, which might lead to potential selection biases and baseline differences in the psychological and pathological conditions of the included patients. Second, we measured their cardiopulmonary fitness using 6MWD instead of maximum oxygen uptake $\left(\mathrm{VO}_{2} \mathrm{max}\right)$, thus 6MWD might not provide a direct fitness assessment. Clinically, 6MWD was suggested as a reliable assessment tool for evaluating physical fitness in the population with schizophrenia [4]. However, future studies employing more rigorous and direct aerobic assessments, such as maximal oxygen uptake or ventilator/lactate threshold intensity, are warranted to better evaluate the improvements in aerobic endurance in population with schizophrenia. Third, because endurance training requires longer washout periods to achieve long-term effects, our exercise intervention cannot be investigated through blind testing or in crossover studies. A final limitation could be that the general control group was not included, but we have to note that the primary purpose of this study was to evaluate the benefit of bench-step training on the fitness and mental state of patients with schizophrenia. Further, because each study included patients who may be using different dosages of different antipsychotic agents, the psychological effects of drug treatment were excluded. We did not include a balance control to accommodate these effects.

\section{Conclusions}

This study demonstrated that the 12-week intervention of moderate-intensity benchstep exercise training (BSET; frequency: 1 session/week; each session of $30 \mathrm{~min}$; step cadence: 96 beats $/ \mathrm{min}$ ) might effectively enhance the aerobic endurance and mood state of patients with chronic schizophrenia. However, their attention did not change after the BSET intervention. We, therefore, suggest that future studies are warranted to conduct 
long-term moderate-intensity exercise interventions and further verify the possible effects of such interventions on improving cognitive functions such as attention.

Author Contributions: Conceptualization, Y.-C.K., D.-Y.C., and Y.-H.L.; methodology, Y.-C.K. and D.-Y.C.; formal analysis, Y.-C.K., D.-Y.C., and Y.-H.L.; investigation, Y.-C.K. and D.-Y.C.; resources, Y.-C.K. and D.-Y.C.; data curation, Y.-C.K. and D.-Y.C.; writing-original draft preparation, Y.-C.K. and Y.-H.L.; writing - review and editing, Y.-C.K. and Y.-H.L.; supervision, Y.-C.K.; project administration, D.-Y.C.; and funding acquisition, Y.-C.K. All authors have read and agreed to the published version of the manuscript.

Funding: This research was funded by the Ministry of Science and Technology (MOST, Taiwan). The APC was partially funded by National Taipei University of Nursing and Health Sciences (NTUNHS) and MOST, Taiwan.

Institutional Review Board Statement: The study was conducted according to the guidelines of the last version of Declaration of Helsinki, and approved by the National Taiwan University Research Ethical Committee (protocol\#2017EM03012, date of approval 9 May 2017).

Informed Consent Statement: Written informed consent was obtained from all participants involved in the study.

Data Availability Statement: The data presented in this study are available upon request from the corresponding author.

Acknowledgments: We sincerely appreciate the efforts from all the participants in this study. The authors thank the National Taipei University of Nursing and Health Sciences for providing us with space, materials, and resources throughout the experimental period.

Conflicts of Interest: The authors declare no conflict of interest.

\section{References}

1. David, A.S. Insight and psychosis. Br. J. Psychiatry 1990, 156, 798-808. [CrossRef]

2. Jablensky, A.; Kirkbride, J.B.; Jones, P.B. Schizophrenia: The epidemiological horizon. In Schizophrenia, 3rd ed.; Weinberger, D.R., Harrison, P., Eds.; John Wiley \& Sons: Hoboken, NJ, USA, 2011; pp. 185-225.

3. van Os, J.; Kenis, G.; Rutten, B.P. The environment and schizophrenia. Nature 2010, 468, 203-212. [CrossRef]

4. Faulkner, G.; Cohn, T.; Remington, G. Validation of a physical activity assessment tool for individuals with schizophrenia. Schizophr. Res. 2006, 82, 225-231. [CrossRef]

5. Beebe, L.H.; Harris, R.F. Description of physical activity in outpatients with schizophrenia spectrum disorders. Int. J. Ment. Health Nurs. 2013, 22, 430-436. [CrossRef] [PubMed]

6. Beebe, L.H.; Tian, L.; Morris, N.; Goodwin, A.; Allen, S.S.; Kuldau, J. Effects of exercise on mental and physical health parameters of persons with schizophrenia. Issues Ment. Health Nurs. 2005, 26, 661-676. [CrossRef] [PubMed]

7. Vancampfort, D.; Probst, M.; Scheewe, T.; Maurissen, K.; Sweers, K.; Knapen, J.; De Hert, M. Lack of physical activity during leisure time contributes to an impaired health related quality of life in patients with schizophrenia. Schizophr. Res. 2011, 129, 122-127. [CrossRef] [PubMed]

8. Tandon, R.; Nasrallah, H.A.; Keshavan, M.S. Schizophrenia, "just the facts" 5. Treatment and prevention. Past, present, and future. Schizophr. Res. 2010, 122, 1-23. [CrossRef]

9. Newcomer, J.W. Metabolic risk during antipsychotic treatment. Clin. Ther. 2004, 26, 1936-1946. [CrossRef]

10. Blouin, M.; Binet, M.; Bouchard, R.H.; Roy, M.A.; Després, J.P.; Alméras, N. Improvement of metabolic risk profile under second-generation antipsychotics: A pilot intervention study. Canadian journal of psychiatry. Can. J. Psychiatry 2009, 54, 275-279. [CrossRef]

11. Wani, R.A.; Dar, M.A.; Margoob, M.A.; Rather, Y.H.; Haq, I.; Shah, M.S. Diabetes mellitus and impaired glucose tolerance in patients with schizophrenia, before and after antipsychotic treatment. J. Neurosci. Rural. Pract. 2015, 6, 17-22. [CrossRef]

12. Holt, R.I.; Peveler, R.C.; Byrne, C.D. Schizophrenia, the metabolic syndrome and diabetes. Diabet. Med. 2004, 21, 515-523. [CrossRef] [PubMed]

13. Henderson, D.C. Schizophrenia and comorbid metabolic disorders. J. Clin. Psychiatry 2005, 66 (Suppl. 6), 11-20.

14. Leucht, S.; Burkard, T.; Henderson, J.; Maj, M.; Sartorius, N. Physical illness and schizophrenia: A review of the literature. Acta Psychiatr. Scand. 2007, 116, 317-333. [CrossRef] [PubMed]

15. De Hert, M.; Schreurs, V.; Vancampfort, D.; Van Winkel, R. Metabolic syndrome in people with schizophrenia: A review. World Psychiatry 2009, 8, 15-22. [CrossRef] [PubMed]

16. Bozikas, V.P.; Kosmidis, M.H.; Kiosseoglou, G.; Karavatos, A. Neuropsychological profile of cognitively impaired patients with schizophrenia. Compr. Psychiatry 2006, 47, 136-143. [CrossRef] 
17. Erickson, K.I.; Prakash, R.S.; Voss, M.W.; Chaddock, L.; Hu, L.; Morris, K.S.; White, S.M.; Wojcicki, T.R.; McAuley, E.; Kramer, A.F. Aerobic fitness is associated with hippocampal volume in elderly humans. Hippocampus 2009, 19, 1030-1039. [CrossRef]

18. Narr, K.L.; Thompson, P.M.; Szeszko, P.; Robinson, D.; Jang, S.; Woods, R.P.; Kim, S.; Hayashi, K.M.; Asunction, D.; Toga, A.W.; et al. Regional specificity of hippocampal volume reductions in first-episode schizophrenia. NeuroImage 2004, 21, 1563-1575. [CrossRef]

19. Bobes, J.; Arango, C.; Garcia-Garcia, M.; Rejas, J. Healthy lifestyle habits and 10-year cardiovascular risk in schizophrenia spectrum disorders: An analysis of the impact of smoking tobacco in the CLAMORS schizophrenia cohort. Schizophr. Res. 2010, 119, 101-109. [CrossRef] [PubMed]

20. Vancampfort, D.; Knapen, J.; Probst, M.; van Winkel, R.; Deckx, S.; Maurissen, K.; Peuskens, J.; De Hert, M. Considering a frame of reference for physical activity research related to the cardiometabolic risk profile in schizophrenia. Psychiatry Res. 2010, 177, 271-279. [CrossRef]

21. Vancampfort, D.; Probst, M.; Sweers, K.; Maurissen, K.; Knapen, J.; De Hert, M. Relationships between obesity, functional exercise capacity, physical activity participation and physical self-perception in people with schizophrenia. Acta Psychiatr. Scand. 2011, 123, 423-430. [CrossRef]

22. Strassnig, M.; Brar, J.S.; Ganguli, R. Nutritional assessment of patients with schizophrenia: A preliminary study. Schizophr. Bull. 2003, 29, 393-397. [CrossRef] [PubMed]

23. Scheewe, T.W.; Backx, F.J.; Takken, T.; Jorg, F.; van Strater, A.C.; Kroes, A.G.; Kahn, R.S.; Cahn, W. Exercise therapy improves mental and physical health in schizophrenia: A randomised controlled trial. Acta Psychiatr. Scand. 2013, 127, 464-473. [CrossRef] [PubMed]

24. Chang, H.; Kim, K.; Jung, Y.-J.; Kato, M. Effects of acute high-Intensity resistance exercise on cognitive function and oxygenation in prefrontal cortex. J. Exerc. Nutr. Biochem. 2017, 21, 1-8. [CrossRef] [PubMed]

25. Kashihara, K.; Maruyama, T.; Murota, M.; Nakahara, Y. Positive effects of acute and moderate physical exercise on cognitive function. J. Physiol. Anthropol. 2009, 28, 155-164. [CrossRef] [PubMed]

26. McMorris, T.; Collard, K.; Corbett, J.; Dicks, M.; Swain, J.P. A test of the catecholamines hypothesis for an acute exercise-cognition interaction. Pharmacol. Biochem. Behav. 2008, 89, 106-115. [CrossRef] [PubMed]

27. McDevitt, J.; Wilbur, J.; Kogan, J.; Briller, J. A walking program for outpatients in psychiatric rehabilitation: Pilot study. Biol. Res. Nurs. 2005, 7, 87-97. [CrossRef]

28. Heggelund, J.; Nilsberg, G.E.; Hoff, J.; Morken, G.; Helgerud, J. Effects of high aerobic intensity training in patients with schizophrenia-A controlled trial. Nord. J. Psychiatry 2011, 65, 269-275. [CrossRef]

29. Kraemer, W.J.; Keuning, M.; Ratamess, N.A.; Volek, J.S.; McCormick, M.; Bush, J.A.; Nindl, B.C.; Gordon, S.E.; Mazzetti, S.A.; Newton, R.U.; et al. Resistance training combined with bench-step aerobics enhances women's health profile. Med. Sci Sports Exerc. 2001, 33, 259-269. [CrossRef] [PubMed]

30. Vancampfort, D.; Probst, M.; Scheewe, T.; Knapen, J.; De Herdt, A.; De Hert, M. The functional exercise capacity is correlated with global functioning in patients with schizophrenia. Acta Psychiatr. Scand. 2012, 125, 382-387. [CrossRef]

31. Marzolini, S.; Jensen, B.; Melville, P.J.M.H.; Activity, P. Feasibility and effects of a group-based resistance and aerobic exercise program for individuals with severe schizophrenia: A multidisciplinary approach. Ment. Health Phys. Act. 2009, 2, 29-36. [CrossRef]

32. Stookey, A.D.; Katzel, L.I.; Steinbrenner, G.; Shaughnessy, M.; Ivey, F.M. The short physical performance battery as a predictor of functional capacity after stroke. J. Stroke Cerebrovasc. Dis. 2014, 23, 130-135. [CrossRef]

33. Smith, A. Symbol Digit Modalities Test; Western Psychological Services: Los Angeles, CA, USA, 1973.

34. Beck, A.T.; Steer, R.A.; Brown, G.K. Manual for the Beck Depression Inventory-II; Psychological Corporation: San Antonio, TX, USA, 1996.

35. van Os, J.; Kapur, S. Schizophrenia. Lancet 2009, 374, 635-645. [CrossRef]

36. Filik, R.; Sipos, A.; Kehoe, P.G.; Burns, T.; Cooper, S.J.; Stevens, H.; Laugharne, R.; Young, G.; Perrington, S.; McKendrick, J.; et al. The cardiovascular and respiratory health of people with schizophrenia. Acta Psychiatr. Scand. 2006, 113, 298-305. [CrossRef]

37. Hasnain, M.; Vieweg, W.V. Do we truly appreciate how difficult it is for patients with schizophrenia to adapt a healthy lifestyle? Acta Psychiatr. Scand. 2011, 123, 409-410. [CrossRef] [PubMed]

38. von Hausswolff-Juhlin, Y.; Bjartveit, M.; Lindstrom, E.; Jones, P. Schizophrenia and physical health problems. Acta Psychiatr. Scand. Suppl. 2009, 119, 15-21. [CrossRef] [PubMed]

39. Tiihonen, J.; Lonnqvist, J.; Wahlbeck, K.; Klaukka, T.; Niskanen, L.; Tanskanen, A.; Haukka, J. 11-year follow-up of mortality in patients with schizophrenia: A population-based cohort study (FIN11 study). Lancet 2009, 374, 620-627. [CrossRef]

40. Koponen, H.; Alaräisänen, A.; Saari, K.; Pelkonen, O.; Huikuri, H.; Raatikainen, M.J.; Savolainen, M.; Isohanni, M. Schizophrenia and sudden cardiac death: A review. Nord. J. Psychiatry 2008, 62, 342-345. [CrossRef]

41. Ruschena, D.; Mullen, P.E.; Burgess, P.; Cordner, S.M.; Barry-Walsh, J.; Drummer, O.H.; Palmer, S.; Browne, C.; Wallace, C. Sudden death in psychiatric patients. Br. J. Psychiatry 1998, 172, 331-336. [CrossRef]

42. Said, M.A.; Sulaiman, A.H.; Habil, M.H.; Das, S.; Bakar, A.K.; Yusoff, R.M.; Loo, T.H.; Bakar, S.A. Metabolic syndrome and cardiovascular risk among patients with schizophrenia receiving antipsychotics in Malaysia. Singapore Med. J. 2012, 53, 801-807.

43. Abidi, O.; Vercherin, P.; Massoubre, C.; Bois, C. The global cardiovascular risk of patients with schizophrenia hospitalized in psychiatry at the university hospital of Saint-Étienne. Encephale 2019, 45, 200-206. [CrossRef] 
44. Vancampfort, D.; Probst, M.; Sweers, K.; Maurissen, K.; Knapen, J.; De Hert, M. Reliability, minimal detectable changes, practice effects and correlates of the 6-min walk test in patients with schizophrenia. Psychiatry Res. 2011, 187, 62-67. [CrossRef]

45. Donaghy, M.E. Exercise can seriously improve your mental health: Fact or fiction? Adv. Physiother 2007, 9, 76-88. [CrossRef]

46. Firth, J.; Cotter, J.; Elliott, R.; French, P.; Yung, A.R. A systematic review and meta-analysis of exercise interventions in schizophrenia patients. Psychol. Med. 2015, 45, 1343-1361. [CrossRef]

47. Malchow, B.; Keeser, D.; Keller, K.; Hasan, A.; Rauchmann, B.S.; Kimura, H.; Schneider-Axmann, T.; Dechent, P.; Gruber, O.; Ertl-Wagner, B.; et al. Effects of endurance training on brain structures in chronic schizophrenia patients and healthy controls. Schizophr. Res. 2016, 173, 182-191. [CrossRef] [PubMed]

48. Kimhy, D.; Lauriola, V.; Bartels, M.N.; Armstrong, H.F.; Vakhrusheva, J.; Ballon, J.S.; Sloan, R.P. Aerobic exercise for cognitive deficits in schizophrenia-The impact of frequency, duration, and fidelity with target training intensity. Schizophr. Res. 2016, 172, 213-215. [CrossRef]

49. Stroth, S.; Hille, K.; Spitzer, M.; Reinhardt, R. Aerobic endurance exercise benefits memory and affect in young adults. Neuropsychol. Rehabil. 2009, 19, 223-243. [CrossRef] [PubMed]

50. Malchow, B.; Keller, K.; Hasan, A.; Dörfler, S.; Schneider-Axmann, T.; Hillmer-Vogel, U.; Honer, W.G.; Schulze, T.G.; Niklas, A.; Wobrock, T.; et al. Effects of endurance training combined with cognitive remediation on everyday functioning, symptoms, and cognition in multiepisode schizophrenia patients. Schizophr. Bull. 2015, 41, 847-858. [CrossRef]

51. Pajonk, F.-G.; Wobrock, T.; Gruber, O.; Scherk, H.; Berner, D.; Kaizl, I.; Kierer, A.; Müller, S.; Oest, M.; Meyer, T.; et al. Hippocampal plasticity in response to exercise in schizophrenia. Arch. Gen. Psychiatry 2010, 67, 133-143. [CrossRef] 